\title{
ON THE MULTI-DIMENSIONAL PARTITIONS OF SMALL INTEGERS
}

\author{
JUN KYO KIM
}

\begin{abstract}
For each dimension exceeds 1, determining the number of multi-dimensional partitions of a positive integer is an open question in combinatorial number theory. For $n \leq 14$ and $d \geq 1$ we derive a formula for the function $\wp_{d}(n)$ where $\wp_{d}(n)$ denotes the number of partitions of $n$ arranged on a $d$-dimensional space. We also give an another definition of the $d$-dimensional partitions using the union of finite number of divisor sets of integers.
\end{abstract}

\section{Introduction}

Partitioning of integers is a problem in number theory dating back to the Middle Ages [3, 2]. A one-dimensional partition of a positive integer $n$ is given by

$$
n=n_{1}+n_{2}+\cdots+n_{k}
$$

where all $n_{i}$ 's are non-negative integers and $n_{i} \geq n_{i+1}$. A two-dimensional or plane partition of an integer is a decomposition into a sum of smaller positive integers which are arranged on a plane. The ordering property generalizes to the summands being non-increasing along both the rows and the columns. Generalization to $d$-dimensional one is straightforward. In defining higherdimensional partitions, the fact that sequences are non-increasing in all the directions becomes important. A $d$-dimensional partition of a positive integer $n$ is an array whose sum is $n$ :

$$
n=\sum_{i_{1}, ., i_{d} \geqslant 0} n_{i_{1} i_{2} \cdots i_{d}}
$$

where the $n_{i_{1} i_{2} \cdots i_{d}}$ are non-negative integers satisfying $n_{i_{1} i_{2} \cdots i_{d}} \geqslant n_{j_{1} j_{2} \cdots j_{d}}$ whenever $i_{1} \leq j_{1}, i_{2} \leq j_{2}, \ldots, i_{d} \leq j_{d}$ [1, p.179]. For example, the following

Received May 3, 2011; Accepted December 16, 2011.

2000 Mathematics Subject Classification. 05A17, 05A18, $11 \mathrm{p} 81$.

Key words and phrases. partitions of integers, multidimensional partition, combinatorial number theory, additive number theory.

This work was supported by a 2-Year Research Grant of Pusan National University. 
is a plane partition of 16 :

$$
\begin{array}{rll}
1 & & \\
4 & 1 \\
5 & 3 & 2 \\
5_{(0,0)}+3_{(0,1)}+2_{(0,2)} & +4_{(1,0)}+1_{(1,1)}+1_{(2,0)}
\end{array}
$$

and the set $\tau=\{(0,0),(0,1),(0,2),(1,0),(1,1),(2,0)\}$ is called a Young diagram of $3+2+1$. See Section 2 for definition and explanation.

We denote $\lambda \vdash_{d} n$ when $\lambda$ is a $d$-dimensional partition of $n$. Let $\wp_{d}(n)$ denote the number of $d$-dimensional partitions of $n$. By convention, let $\wp_{d}(0)=1$. For example, $\wp_{2}(3)=6$ since there are six plane partitions with sum 3 :

$$
\begin{array}{llllllllll}
1 & 1 & 1 & 1 & & 1 & 1 & & & \\
& 1 & 1 & 2 & 1 & 2 & 3 .
\end{array}
$$

In Section 2, we give another definition of the function $\wp_{d}(n)$. Section 3 describes how to get the values of the function $\wp_{d}(n)$ for integers $n$ up to 14 .

\section{Higher-dimensional partitions}

A one-dimensional partitions can be graphically visualized with Young diagrams $[7,6]$. A Young diagram (also called Ferrers diagram) is a finite collection of cells arranged in left-justified rows, with the row lengths weakly decreasing. Listing the number of boxes in each row gives a partition of a non-negative integer $n$. For a Young diagram $\nu$, let $|\nu|$ be the total number of cells of the diagram. Young diagrams will be drawn using the French notation with the longest row on the bottom and will be identified with the partition itself by referring to a partition as a collection of cells. For example, the Young diagrams corresponding to the partitions of 4 are

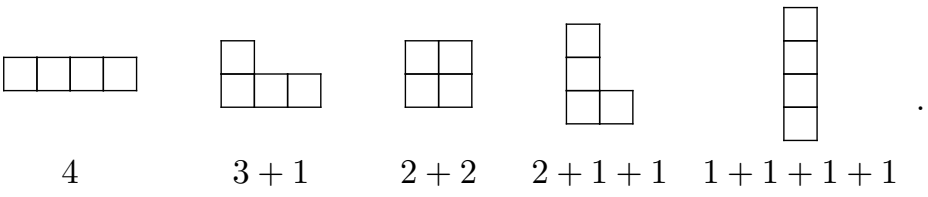

Since there is a obvious one-to-one correspondence between one-dimensional partitions and Young diagrams, we use these two terms interchangeably.

Let $\mathbb{N}$ be the set of all non-negative integers, $\mathbb{N}^{*}$ be the set of all positive integers and $p_{i}$ be the $i$ th prime(i.e., $p_{1}=2, p_{2}=3$, etc.). For $n \in N^{*}$, let $D V(n)$ be the set of all positive divisors of $n$ and $\omega(n)$ be the largest prime factor of $n$.

Definition 1. Let $d$ and $n$ be positive integers. Then we define a function

$$
H_{d}(n)=\left\{s \in \mathcal{H}_{d}|| s \mid=n\right\},
$$


where

$$
\mathcal{H}_{d}=\left\{\bigcup_{\ell=1}^{k} D V\left(n_{\ell}\right) \mid n_{\ell} \text { 's are positive integers with } \omega\left(n_{\ell}\right) \leq p_{d+1}\right\} .
$$

For example, $\left|H_{2}(3)\right|=6$ since

$$
H_{2}(3)=\left\{\left\{1,2,2^{2}\right\},\left\{1,3,3^{2}\right\},\left\{1,5,5^{2}\right\},\{1,2,3\},\{1,2,5\},\{1,3,5\}\right\} .
$$

Let $n$ be a positive integer and $\nu$ be any Young diagram with $n$ boxes. Because any cell $(i, j)$ in $\nu$ can be filled with the number $2^{j} 3^{i}$, we know that for any left side of or below of the cell $(i, j)$ are filled with divisors of $2^{j} 3^{i}$. Thus, we have

Consequently,

$$
\bigcup_{(i, j) \in \nu}\left\{2^{j} 3^{i}\right\}=\bigcup_{(i, j) \in \nu} D V\left(2^{j} 3^{i}\right) \in H_{1}(n)
$$

$$
n=\left|\bigcup_{(i, j) \in \nu} D V\left(2^{j} 3^{i}\right)\right| .
$$

Conversely, if $A$ is an element of $H_{1}(t)$, then the set

$$
\widetilde{A}=\left\{\operatorname{cell}(i, j) \mid(i, j) \in \mathbb{N}^{*} \times \mathbb{N}^{*} \text { and } 2^{j} 3^{i} \in A\right\}
$$

is a Young diagram with $t$ boxes.

By the obvious one-to-one correspondence above, we have

Proposition 2.1. Let $n$ be a positive integer. Then

$$
\wp_{1}(n)=\left|H_{1}(n)\right| .
$$

For example, $\left|H_{1}(4)\right|=5$ since



Theorem 2.2. For a positive integer $d$, we have

$$
\wp_{d}(n)=\left|H_{d}(n)\right| \text { for all } n \in \mathbb{N}^{*} .
$$

Proof. Let

$$
\lambda=\left[\sum_{i_{1}, ., i_{d} \geqslant 0} n_{i_{1} i_{2} \cdots i_{d}}\right]
$$

be a $d$-dimensional partition of a positive integer $n$. The $d$-dimensional partition $\lambda$ may be reinterpreted to

$$
\hat{\lambda}=\left[\sum_{\omega(t) \leq p_{d}} n_{t}\right]
$$


where the $n_{t}$ 's are positive integers satisfying $n_{t} \geq n_{s}$ whenever $t$ divides $s$. Then we write $\hat{\lambda} \hat{\vdash}_{d} n$.

For a set $S$, let $a S=\{a s \mid s \in S\}$. From (2.1) and the definition of the function $D V$, we obtain

$$
\begin{aligned}
& n=\sum_{\omega(t) \leq p_{d}}\left|t D V\left(p_{d+1}^{n_{t}-1}\right)\right| \\
& n_{v} \geq n_{s} \text { whenever } v \mid s \\
& =\left|\begin{array}{c}
\bigcup_{\omega(t) \leq p_{d}} t D V\left(p_{d+1}^{n_{t}-1}\right) \\
n_{v} \geq n_{s} \text { whenever } v \mid s
\end{array}\right| \\
& =\left|\bigcup_{\omega(t) \leq p_{d}} D V\left(t p_{d+1}^{n_{t}-1}\right)\right|,
\end{aligned}
$$

which proves the theorem.

Lemma 2.3. Let $d$ be a positive integer. Then

$$
\wp_{d}(n)=\left|\left\{\left[1_{t_{1}}+\cdots+1_{t_{n}}\right] \hat{\vdash}_{d+1} n\right\}\right| .
$$

Proof. From Theorem 2.2 and (2.2), we have

$$
\begin{aligned}
\left|H_{d}(n)\right| & =\left|\left\{\tau=\bigcup_{\substack{\omega(t) \leq p_{d} \\
n_{v} \geq n_{s} \text { whenever } v \mid s}} D V\left(t p_{d+2}^{1-1}\right)|| \tau \mid=n\right\}\right| \\
& =\left|\left\{\left[\sum_{\omega(t) \leq p_{d}} 1_{t}\right] \hat{\vdash}_{d+1} n\right\}\right| \\
& =\left|\left\{\left[1_{t_{1}}+\cdots+1_{t_{n}}\right] \hat{\vdash}_{d+1} n\right\}\right| .
\end{aligned}
$$

To evaluate $\wp_{d}(2)$, one can easily see that the number of $d$-dimensional partitions of 2 is $d+1$ since

$$
\wp_{d}(2)=\left|H_{d}(2)\right|=\left|\left\{\left\{1, p_{i}\right\} \mid i=1, \ldots, d+1\right\}\right|=d+1 .
$$

By the following theorem, the number of $d$-dimensional partitions of each integer less than 7 can be evaluated. 
Theorem 2.4. ([5], [1] Theorem 11.8.)

$$
\begin{aligned}
& \wp_{d}(0)=1, \\
& \wp_{d}(1)=1, \\
& \wp_{d}(2)=\left(\begin{array}{l}
d \\
1
\end{array}\right)+1, \\
& \wp_{d}(3)=1+2 d+\left(\begin{array}{c}
d \\
2
\end{array}\right), \\
& \wp_{d}(4)=1+4 d+4\left(\begin{array}{c}
d \\
2
\end{array}\right)+\left(\begin{array}{c}
d \\
3
\end{array}\right), \\
& \wp_{d}(5)=1+6 d+11\left(\begin{array}{c}
d \\
2
\end{array}\right)+7\left(\begin{array}{l}
d \\
3
\end{array}\right)+\left(\begin{array}{l}
d \\
4
\end{array}\right), \\
& \wp_{d}(6)=1+10 d+27\left(\begin{array}{l}
d \\
2
\end{array}\right)+28\left(\begin{array}{l}
d \\
3
\end{array}\right)+11\left(\begin{array}{l}
d \\
4
\end{array}\right)+\left(\begin{array}{l}
d \\
5
\end{array}\right) .
\end{aligned}
$$

Since the set $H_{d}(n)$ is fixed by any permutation of $\left\{p_{1}, \ldots, p_{d+1}\right\}$, we have

Lemma 2.5. Let $n$ be a fixed positive integer greater than 1 . Then there exist non-negative integers $a(n, i)$ which satisfy the following:

$$
\wp_{d}(n)=\sum_{i=1}^{n-1} a(n, i)\left(\begin{array}{c}
d+1 \\
i
\end{array}\right)
$$

Proof. Let $\chi(m)$ be the set of all prime factors of $m$. For a positive $i$, define

$$
G_{d}(n, i)=\left\{\left[1_{t_{1}}+\cdots+1_{t_{n}}\right] \hat{\vdash}_{d+1} n \mid \chi\left(\prod_{j=1}^{n} t_{j}\right)=\left\{p_{1}, \ldots, p_{i}\right\}\right\} .
$$

Then by Lemma 2.3,

$$
\begin{aligned}
& \left|\left\{\left[1_{t_{1}}+\cdots+1_{t_{n}}\right] \hat{\vdash}_{d+1} n\right\}\right| \\
= & \left|\bigcup_{i=1}^{n-1}\left\{\left[1_{t_{1}}+\cdots+1_{t_{n}}\right] \hat{\vdash}_{d+1} n|| \chi\left(\prod_{j=1}^{n} t_{j}\right) \mid=i\right\}\right| \\
= & \sum_{i=1}^{n-1}\left|G_{d}(n, i)\right|\left(\begin{array}{c}
d+1 \\
i
\end{array}\right) .
\end{aligned}
$$

which is the result what we want.

We calculated (by computer) $a(n, i)$ for each of $n \leq 14$ which are defined on Lemma 2.5. Thus, we have 
Theorem 2.6. Let $d$ be a positive integer.

$$
\begin{aligned}
\wp_{d}(2) & =[[1]]_{d}, \\
\wp_{d}(3) & =[[1,1]]_{d}, \\
\wp_{d}(4) & =[[1,3,1]]_{d}, \\
\wp_{d}(5) & =[[1,5,6,1]]_{d}, \\
\wp_{d}(6) & =[[1,9,18,10,1]]_{d}, \\
\wp_{d}(7) & =[[1,13,44,49,15,1]]_{d}, \\
\wp_{d}(8) & =[[1,20,97,172,110,21,1]]_{d}, \\
\wp_{d}(9) & =[[1,28,195,512,550,216,28,1]]_{d}, \\
\wp_{d}(10) & =[[1,40,377,1370,2195,1486,385,36,1]]_{d}, \\
\wp_{d}(11) & =[[1,54,694,3396,7603,7886,3514,638,45,1]]_{d}, \\
\wp_{d}(12) & =[[1,75,1251,7968,23860,35115,24318,7484,999,55,1]]_{d}, \\
\wp_{d}(13) & =[[1,99,2185,17910,69580,138155,138075,65997,14667, \\
1495,66,1]]_{d} & \\
\wp_{d}(14) & =[[1,133,3765,38942,191795,495870,677663,471276, \\
1 & 161202,26875,2156,78,1]]_{d},
\end{aligned}
$$

where $\left[\left[c_{1}, \ldots, c_{t}\right]\right]_{d}=\sum_{i=1}^{t} c_{i}\left(\begin{array}{c}d+1 \\ i\end{array}\right)$.

\section{Concluding remarks and exact enumeration}

Previous attempts on studying solid partitions with a computer have been based on exact enumeration [5, 4]. Lemma 2.3 have been based on exact enumeration and the algorithm is made by the following. Partitions that are related to each other by symmetry operations are counted only once and multiplied by the corresponding symmetry factor. In Table 1, we list the number of $d$-dimensional partitions from $n=1$ to $n=9$ for each $d=2,3, \ldots, 9$.

TABLE 1. The number of multi-dimensional partitions of $n$.

\begin{tabular}{|r|r|r|r|r|r|r|r|r|r|}
\hline & $\wp_{1}(n)$ & $\wp_{2}(n)$ & $\wp_{3}(n)$ & $\wp_{4}(n)$ & $\wp_{5}(n)$ & $\wp_{6}(n)$ & $\wp_{7}(n)$ & $\wp_{8}(n)$ & $\wp_{9}(n)$ \\
\hline 1 & 1 & 1 & 1 & 1 & 1 & 1 & 1 & 1 & 1 \\
\hline 2 & 2 & 3 & 4 & 5 & 6 & 7 & 8 & 9 & 10 \\
\hline 3 & 3 & 6 & 10 & 15 & 21 & 28 & 36 & 45 & 55 \\
\hline 4 & 5 & 13 & 26 & 45 & 71 & 105 & 148 & 201 & 265 \\
\hline 5 & 7 & 24 & 59 & 120 & 216 & 357 & 554 & 819 & 1165 \\
\hline 6 & 11 & 48 & 140 & 326 & 657 & 1197 & 2024 & 3231 & 4927 \\
\hline 7 & 15 & 86 & 307 & 835 & 1907 & 3857 & 7134 & 12321 & 20155 \\
\hline 8 & 22 & 160 & 684 & 2145 & 5507 & 12300 & 24796 & 46209 & 80920 \\
\hline 9 & 30 & 282 & 1464 & 5345 & 15522 & 38430 & 84625 & 170370 & 319555 \\
\hline 10 & 42 & 500 & 3122 & 13220 & 43352 & 118874 & 285784 & 621316 & 1247779 \\
\hline
\end{tabular}


A finite sequence of real numbers $\left\{b_{0}, b_{1}, \ldots, b_{m}\right\}$ is said to be unimodal if there exists an index $0 \leq m^{*} \leq m$, called the mode of the sequence, such that $b_{j}$ increases up to $j=m^{*}$ and decreases from then on. We finish this article by noting that Theorem 2.6 indicates that the sequences $\{a(n, i)\}_{i=1, \ldots, n-1}$, which are defined in Lemma 2.5, are unimodal for $n=1, \ldots, 14$. Therefore, we now mention an additional problem.

Are the sequences $\{a(n, i)\}_{i=1, \ldots, n-1}$, which are defined in Lemma 2.5 , unimodal for all $n$ ?

\section{References}

[1] G. E. Andrews, The Theory of Partitions, Reading, Massachusetts: Addison-Wesley Publishing Company, 1976.

[2] G. H. Hardy and E. M. Wright, An Introduction to the Theory of Numbers 4 th edn Oxford: Clarendon, 1960.

[3] L. Euler, De partitione numerorum, Novi Commentarii Academiae scientiarum Petropolitanae, 3 (1753), 125-169.

[4] F. Wang and D. P. Landau, Multiple-range random walk algorithm to calculate the density of states, Physical Review Letters - PHYS REV LETT, 86, no 10 (2001), 20502053.

[5] A. O. L. Atkin, P. Bratley and I. G. Macdonald, Some computations for m-dimensional partitions, Proc. Camb. Phil. Soc. 63(4) (1967), 1097-1100.

[6] W. Fulton, Young tableaux. With applications to representation theory and geometry, London Mathematical Society Student Texts 35 (1997), Cambridge University Press, Cambridge.

[7] A. Yong, What is... a Young tableau?, Notices Amer. Math. Soc. 54 (2007), 240-241.

JUN KYO KIM

Department of Mathematics, Pusan National University, Pusan 609-735, Korea

E-mail address: junkyo@pusan.ac.kr 